\title{
L'Exposition Internationale de la Houille Blanche et du Tourisme
}

\author{
par Victor Sylvestre, Ingénieur A. M. et I. E. G., \\ Secrélaire de la Classe VI "Gros matériel électrique » de l'Exposition Internationale de la Houille Blanche et du Tourisme.
}

"Ainsi une grande révolution est accomplie; la montagne, jusque-là inactive et inféconde, va pren"dre part au labeur universel; elle est domptée et maitrisée par son propre fils; le montagnard".

Gabriel Hanotalx,

'Congrès de la Houille Blanche, en 1902).

Avant d'entrer dans le sujet qui nous occupe aujourd'hui : de l'Exposition Internationale de la Houille Blanche et du Tourisme qui vient de s'ouvrir à Grenoble, le 21 Mai, et du troisième Congrès de la Houille Blanche qui aura lieu en juillet prochain, nous tenons tout particulièrement, résumer en quelques mots, l'historique de la houille blanche (1).

L'emploi du moteur hydraulique remonte à la plus haute antiquité. De bonne heure l'homme sut employer la puissance des eaux courantes pour actionner des roues ou rouets en bois lui permettant de moudre son blé, scier son bois ou forger ses outils. Ses besoins étaient alors modestes et pendant longtemps les puissances mises en jeu furent faibles : quelques chevaux seulement. Puis, il employa la force des torrents à l'élévation des eaux, afin d'irriguer les cultures.

C'est à M. Aristide Bergès qu'est due cette belle expression de "Houille Blanche \%. Dans une note parue en 1889 , à l'occasion del'Exposition Universelle il dit, en effet:

"Il semble que le moin" dre filet d'eau, dans les " grandes hauteurs, n'est " plus de l'eau, mais de la houille noire qui sourd automati"quement du sol et alors le nom de "Houille Blanche" pour " baptiser ces richesses, vient naturellement à l'esprit.

"De la " Houille Blanche "; dans tout cela, il n'y en a pas, ce " n'est évidemment qu'une métaphore, mais j'ai voulu employer " ce mot pour frapper l'imagination et signaler avec vivacité "que les glaciers et les montagnes peuvent être exploitées en " forces motrices, être pour la région et pour l'Etat, des richesses " aussi précieuses que la houille des profondeurs.

En 1837. - Le Stéphanois Fourneyron aménage dans le Grand Duché de Bade, une chute de 112 mètres.

Vers 1850. - Le François Girard installe des turbines près de Gênes, en Italie.

En 1863. - M. Joya père, équipe chez MM. VICAT \& C $\mathrm{C}^{\mathrm{ie}}$, à Uriage, une chute de 80 mètres; cette chute, qui fonctionne encore, est la plus ancienne en Dauphiné et probablement en France.

(1) Voir l'ouvrage La Houllle Blanche, V. Sylvestre, in vente chez Rey, Grande-Rue, à Grenoble, à un prix exceptionnel à l'occasion de l'Exposition.
En 1864. - M. A. Matessière vient se fixer à Domène où il crée en 1866-1867 la chute de 30 mètres aux Gorges.

En 1867. - M. Aristide Bergès, sous les conseils de II. MatusSrÈnE, vient installer à la Papeterie des Corges un nouveau type de défibreur à pression hydraulique. A la même époque, M. Alfred Freder vient exploiter la papeterie du Moustier à Domène.

En 1868. - M. A. Bergès se fixe en Dauphiné, à Lancey, et installe, en collaboration avec le docteur MARMONNIER, de Domène, la chute de 200 mètres.

En 1868. - M. A. Fredet et M. A. MAtussière associent dans le but d'équiper la chute de 137 m. de Brignoud.

C'est entre 1864 et 1873 , que naquirent et se développèrent, au milieu de bien des vicissitudes, ces papeteries échelonnées le long de la belle vallée du Grésivaudan : à Domène, à Lancey, à Brignoud, Pontcharra, etc.., au débouché de tous les petits torrents qui descendent du massif de Belledonne et se jettent dans l'Isère.

En 1870. - Gramme invente la machine dynamo-électrique.

En 1873. - A l'Exposition de Vienne, l'ingénieur français H. Fontaine révèle la marche en moteur d'une dynamo à courant continu.

En 1880. - L'ingénieur américain PeLtor construit ses premières turbines tangentielles hélicoïdes.

En 1881. - A lieu, à Paris, la première Exposition Internationale d'électricité.

En 1882. - M. A. Bergès porte sa cluute de Lancey à 500 m., et aménage un siphon dans le lac Crozet.

De 1882 à 1886. - M. Marcel Deprez fait un certain nombre d'expériences dans le but de vérifier les lois de la transmission de l'énergie électrique et, en particulier, en 1883, il exécute, sur la demande de la municipalité grenobloise, la célèbre expérience du transport d'énergie de Vizille à Grenoble.

En 1889. - M. Aubry équipe la chute du Venthon, en Savoie, et, en 1892, il songe à utiliser le lac de la Girotte, afin de régulariser le débit du Doron. 
En 1889. - L'Exposition Universelle de Paris, consacre les applications de l'électricité.

En 1891. - Installation du transport, en courant triphasé, 25.000 volts, de Lauffen à Francfort.

En 1898. - M. Dusauger met au point le premier, en France, transport à courant triphasé, 15.000 volts, entre Engins et Voiron.

En 1899. - La Société Fure \& Morge \& Vizille, inaugure, sous la direction de M. LÉPINE, la centrale hydroélectrique de Champ, sur le Drac, première usine importante de la région des Alpes.

L'industrie de la Houille Blanche était née.

En 1900. - Fondation de l'Institut Electrotechnique de Grenoble.

En 1901. - Création à Grenoble du Syndicat des Forces Hydrauliquès.

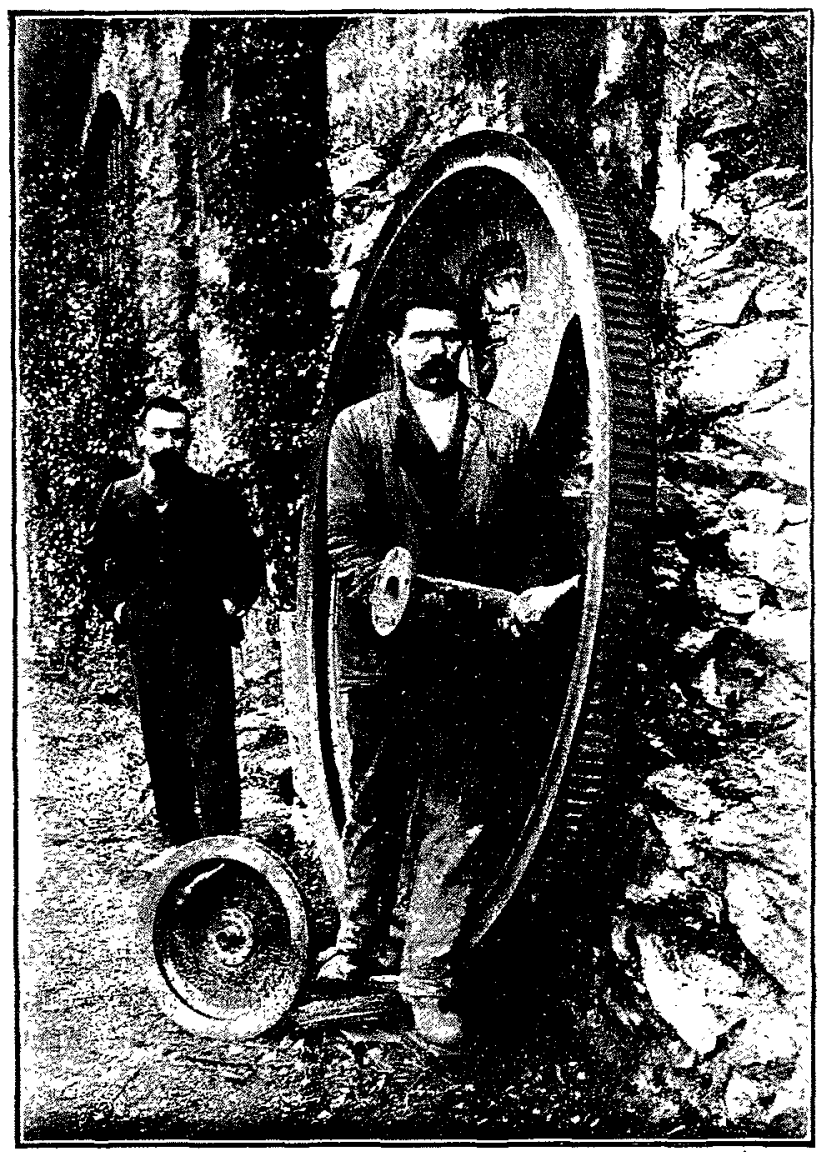

Une des premières roues de turbine de la chute de Lancey, équipée par M. Aristide Bergès

En 1902. - Le premier Congrès de la Houille Blanche a lieu à Grenoble, sous la présidence de $M$. Charles Pinat, et sous la présidence d'hónneur de MM. Guillain et Gabriel Hanotaux.

En 1903. - La Société de Fure \& Morge \& Vizille porte la tension de son réseau à 26.000 volts " GrenobleVoiron ", premier transport à 26.000 volts en France.

En 1906. - La Société Générale de Force \& Lumrère' installe la ligne Moûtiers-Lyon, à courant continu série, à la tension remarquable de 55.000 volts.

En 1910. - Mise sous tension de la ligne triphasée 60.000 volts de Grenoble, St-Chamond, longueur : 117 kilom.
En 1922. - Mise en marche de l'usine de Beaumont-Monteux (Basse-Isère) et de la ligne 120.000 volts allant sur St-Etienne.

En 1924. - La Compagnie des Chemins de fer du Midi met en fonctionnement ses lignes 150.000 volts pour l'alimentation des chemins de fer.

En 1925. - Mise en marche de l'usine de la VraLAine, à Ste-Foy (Hante-Isère), et de la ligne 120.000 volts : La Viclaire-Lyon.

- Exposition Internationale de Ia Houille Blanche ef du Tourisme à Grenoble et Congrès de la Houille Blanche, montrant au monde le chemin parcouru et les progrès réalisés depuis un demi-siècle dans la technique de l'équipement des chutes et des transports d'énergie.

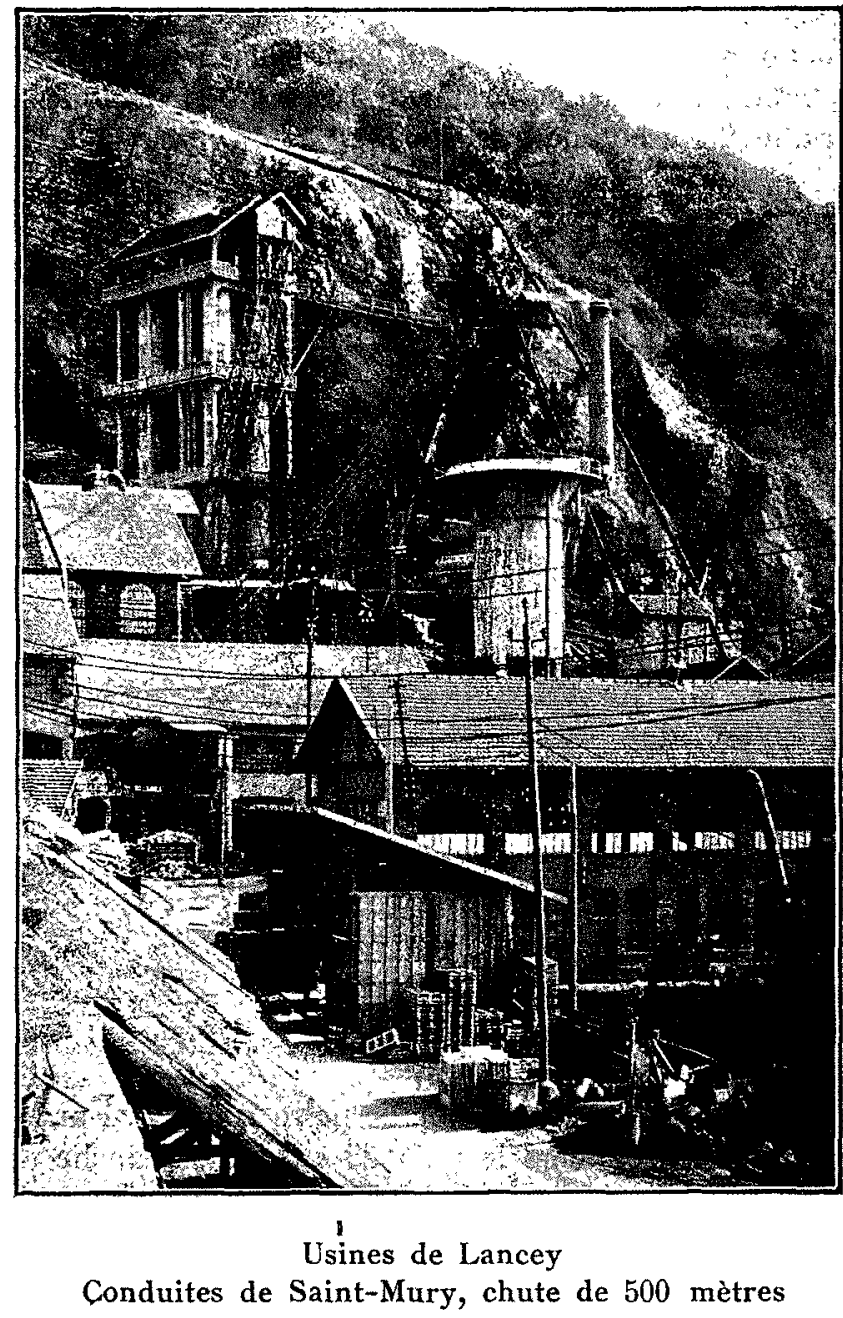

La puissance totale aménagée de nos chutes d'eau, qui était en 1914 d'environ 750.000 chevaux, s'est augmentée de 850.000 chevaux pendant la guerre; en tenant compte des usines hydroélectriques qui ont été mises en fonctionnement depuis, on peul estimer actuellement à environ 2.500 .000 chevaux la puissance totale aménagée sur nos torrents et rivières de France.

« Ce n'est donc pas la douce France qui s'est laissée vivre, comme on le lui reproche à satiété, c'est la France forte qui a livré la bataille et emporté le succès. "

Comme l'a dit M. Raoul Branchard, en vérité c'est une belle œuvre qui a été accomplie depuis un demi-siècle le long des cours d'eau de notre France. Ne craignons pas de le dire, car elle fait honneur aux industriels qui n'ont pas hésité à se lancer dans ces entreprises nouvelles, et l'ont fait sans timidité, voyant grand 
du premier coup. Elle fait honneur aux Ingénieurs qui ont marché à la tête du progrès, n'oublions pas que ce sont des Français, installés dans les Alpes : les Bergès, les Freder, les Matussiène, les Joya, les Aubry, etc., qui ont été les premiers à utiliser la Houille Blanche, et que ce sont encore des Français, H. Fontaine M. Derrez, etc., qui ont trouvé et développé les applications des transports d'énergie électrique.

\section{L'EXPOSITION INTERNATIONALE DE LA HOUILLE BLANCHE}

\section{ET DU TOURISME}

Le Conseil Général de l'Isère, la Ville et la Chambre dé Commerce de Grenoble, ont décidé d'organiser dans cette ville, en 1925, une Exposition Internationale de la Houille Blanche et du Tourisme. L'année 1925 a été choisie pour cette manifestation, ger, dans le domaine de la houille blanche, envisagé non seulement au point de vue de la production et de la distribution de l'énergie électrique, mais encore au point de vue de toutes les applications de l'électricité puis dans le domaine du tourisme proprement dit, de l'hôtellerie, et de toutes les industries touristiques ;

$2^{\circ}$ En mesurant ainsi ce qui a été fait, d'évaluer ce qui peut rester encore à faire ;

$3^{\circ}$ De stimuler, par la comparaison des méthodes employées et des résultats obtenus, toutes les initiatives fécondes, toutes les énergies créatrices, en faveur de ces deux grandes branches de l'activité humaine qui tendent de plus en plus à prendre une place prépondérante dans toutes les nations civilisées ;

$4^{\circ}$ Enfin, de hâter, par ce moyen, dans toute la mesure du possible, aussi bien dans l'ordre scientifique que dans l'ordre

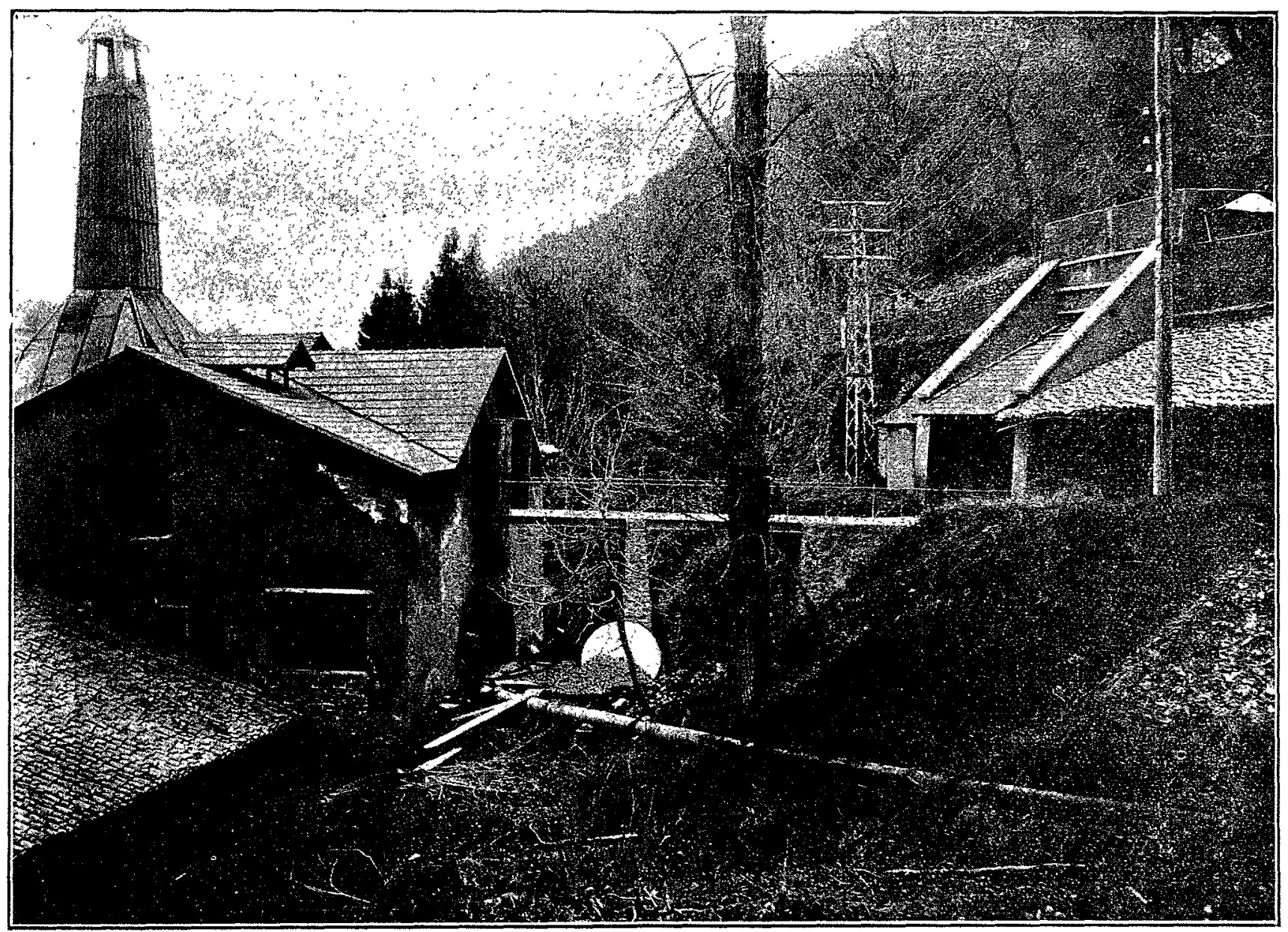

Usine à ciments d'Uriage-les-Bains (Isère)

Conduite construite en 1863 - Diamètre de la conduite : $0 \mathrm{~m}$. 300 - Longueur : $460 \mathrm{~m}$. - Hauteur de chute : $110 \mathrm{~m}$. - Puissance : 112 HP

afin de devancer l'Italie qui se proposait d'ouvrir, en 1926, à Turin, une Exposition analogue. Cette manifestation a obtenu immédiatement le patronage officiel du Gouvernement français qui l'a accréditée officiellement auprès des Gouvernements amis de la France et qui lui a, en outre, accordé une importante subvention.

D'autre part les ministères intéressés, et notamment le Ministère des Travaux Publics, le Ministère du Commerce et de l'Industrie, le Ministère des Colonies, le Ministère de l'Agriculture, etc......, apportent à cette manifestation leur plus entier et leur plus efficace concours.

L'Exposition Internationale de la Houille Blanche et du Tourisme a pour but :

$1^{\circ}$ De bien mettre en lumière tous les immenses progrès accomplis au cours de ces dernières années, tant en France qu'à l'étran- économique, industriel et touristique, la réalisation rapide de nouveaux et décisifs progrès.

Il appartenait à la ville de Grenoble d'être choisie comme siège d'une Exposition Internationale de la Houille Blanche et du Tourisme.

Dans l'ordre de la Houille Blanche, c'est en effet dans la belle vallée du Grésivaudan, à côté de Grenoble, à Brignoud, à Lancey, à Domène, que, dès 1869, les Aristide Bergès, les Fredert, les MAtussière, etc..., ces audacieux pionniers de la houille blanche, installaient les premières hautes chutes. C'est encore entre Grenoble et Vizille que Marcel Deprez, en 1883, se livrait à des expériences décisives sur le transport de l'énergie à distance par le courant électrique (1).

(1) Voir l'historique complet de la Houille Blanche et des Transports d'énergie dans l'ouvrage La Houille Blanche, par V. Sylvestre, en vente à la librairie Rey, Grande-Rue, à Grenoble. 
C'est toujours dans les environs de Grenoble que se créèrent, dès la fin du siècle dernier, les premières puissantes centrales hydrauliques produisant des milliers de chevaux utilisés sur place ou transportés à des centaines de kilomètres pour alimenter en force motrice les industries les plus diverses et éclairer les villes et les campagnes.

Dans l'ordre touristique, Grenoble, capitale des Alpes françaises, se troure placée au seuil même de l'admirable et formidable massif qui, du lac de Genève à la Méditerranée, constitue notre frontière du Sud-Est. Cette situation privilégiée fait de Grenoble un véritable centre distributeur des milliers de touristes qui viennent chaque année séjourner dans les Alpes ou les visiter ; elle tend aussi de plus en plus à faire de Grenoble un centre très important de sports d'hiver.

Pour ces raisons, Grenoble se trouve placée à la fois :

$1^{0}$ Sur la route d'été des Alpes que la Compagnie P.-L.-M. a
ORGANISATION ADMINISTRATIVE DE L'EXPOSITION.

Cette organisation comprend

a) Un Conseil supérieur, composé de trois représentants de chacune des trois collectivités : Conseil Général de l'Isère, Ville et Chambre de Commerce de Grenoble, qui ont pris l'initiative de l'entreprise et qui en assument la responsabilité.

b) Un Commissaire général nommé par décret présidentiel en date du 29 décembre 1923.

c) Trois Commissaires généraux adjoints tenant leurs pouvoirs du Commissaire général, attachés plus spécialemenl, l'un à la Houille Blanche, l'autre à la section Tourisme, et le troisième à tout ce qui se rattache à la participation étrangère.

d) Un Secrétariat général pour l'exécution des décisions prises et la direction des Services de gestion et d'exploitation.

e) Une Section technique complète.

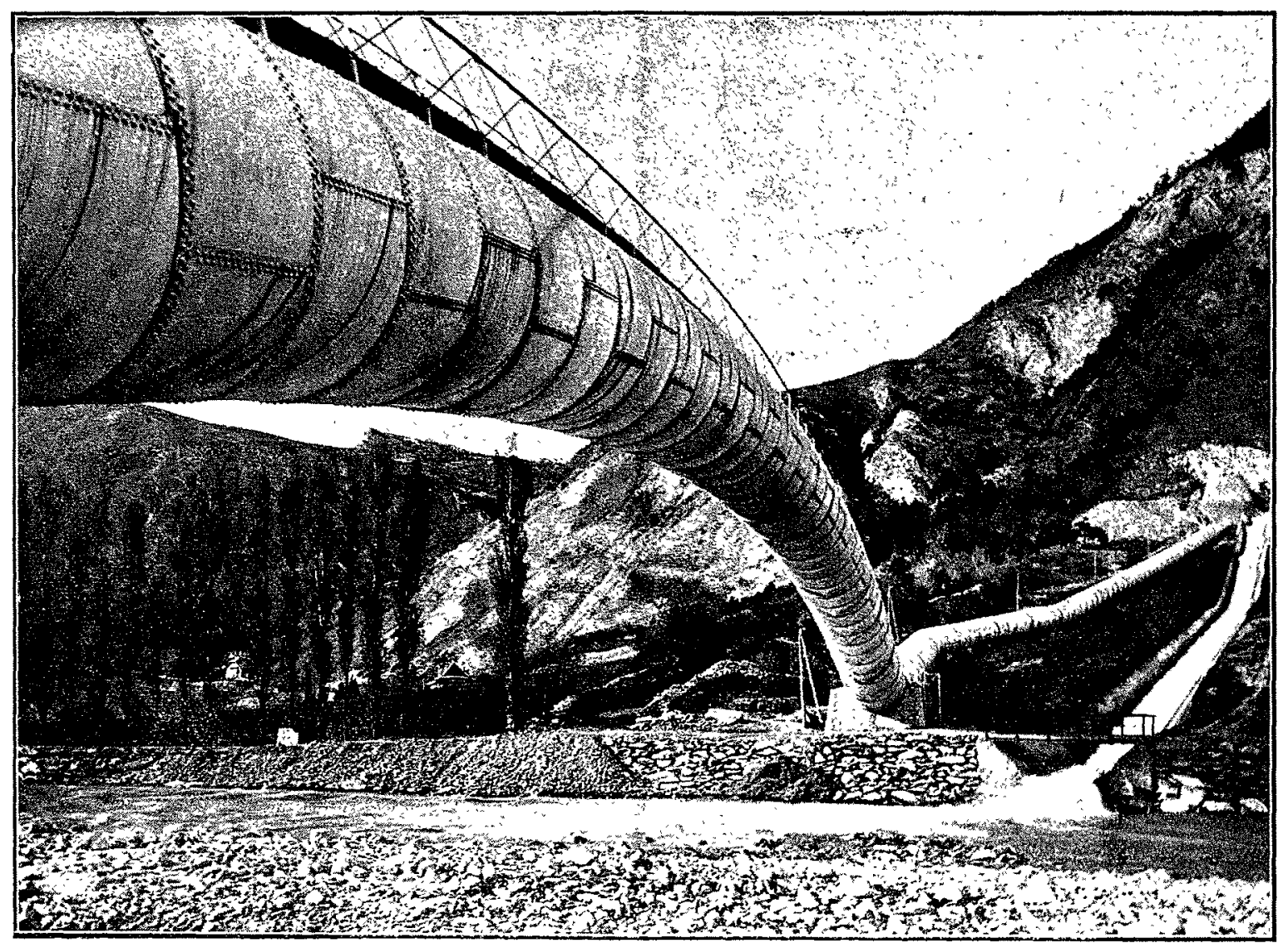

Traversée-en-arc-d'une-rivière par une-conduite forcée - Usine de Saint-Jean-de-Maurienne (Savoie)

mis en exploitation régulière, et avec un succès toujours croissant, depuis plusieurs années;

$2^{\circ}$ Sur la route d'hiver de ces mêmés Alpes, qui permettra de se rendre directement en automobile, en plein hiver, de Paris à Nice par Grenoble, et les massifs neigeux alpins, dont le tracé et l'aménagement ont été mis à l'étude par le Touring-Club de France, le Club Alpin Français, avec le concours de la Compagnie P.-L.-M.

C'est aussi à Grenoble qu'ont été créés :

En 1899. - Le premier Syndicat d'initiative qui ait fonctionné en France, ouvrant ainsi une voie féconde aux cinq ou six cents organisations similaires qui se sont fondées depuis,

En 1900. - L'Institut Electrotechnique, par MM. JANET et PIONCHON.

En 1901. - Le Syndicat des Forces hydrauliques.

En 1902. - Le premier Congrès de Ia houille blanche. f) Des Commissions spécialisées à la propagande, à la publicité, au logement, au Contentieux, etc... Et enfin.

g) Au-dessus de cette solide ossature, se trouvent un Comité d'honneur, un Comité supérieur de patronage et un Comité technique d'organisation, au sein duquel seront constitués les groupes et classes, ainsi que le Jury.

\section{Conseil supérieur.}

Délégués du Conseil municipal de Grenoble: MM. Paul Mistral, député de l'Isère, conseiller général de l'Isère, maire de Grenoble ; Adrien Ricard, négociant, adjoint au maire de Grenoble; Paul Cocat, avocat à la Cour d'appel, vice-président de la Commission administrative des Hôpitaux civils de Grenoble, conseiller municipal.

Délégués du Conseil général de l'Isère : MM. Léon PERRIER, sénateur, président du Conseil général de l'Isère à Grenoble; Dr L. Martin, pharmacien, professeur à l'Ecole de médecine et 
de pharmacie de Grenoble, Conseiller général ; Richard-BerenGER, propriétaire à Mens (Isère), Conseiller général.

Délégués de la Chambre de Commerce de Grenoble: MM. Charles LÉPINE, président de la Chambre de Commerce, ingénieur des Arts et Manufactures, administrateur-délégué de la Société Hydro-électrique de Fure et Morge et Vizille, à Grenoble ; Charles Keller, vice-président de la Chambre de Commerce, industriel électrométallurgiste à Livet (Isère); Aimé Bouchayer, viceprésident de la Chambre de Commerce, industriel à Grenoble.

\section{CoMmISSARIAT GÉNÉRAL.}

Commissaire général : M. Marius Blanchet, industriel, membre de la Chambre de Commerce de Grenoble.

Commissaires généraux adjoints : Section de la Houille Blanche, M. Georges Flusin, professeur d'électrochimie et d'électrométallurgie à la Faculté des Sciences de Grenoble. - Section du nement, professeur à l'Ecole Nationale Supérieure des BeauxArts, à Paris.

Secrétaire administratif : M. Charles MARÉchal, ingénieur, ancien directeur de réseaux de tramways et de distribution d'énergie.

\section{ORgANISATION FINANCIÈRE DE L'EXPOSITION.}

Elle repose sur les bases suivantes :

a) Les trois collectivités: Ville de Grenoble, Département de l'Isère et Chambre de Commerce, qui ont la responsabilité de la manifestation, se sont engagées à verser solidairement une somme minimum de trois millions.

b) Le Commissariat général a organisé une émission de bons à lots pour un capital de neuf millions de francs, qui laissera disponible, en faveur de l'Exposition, une somme de cinq millions environ.

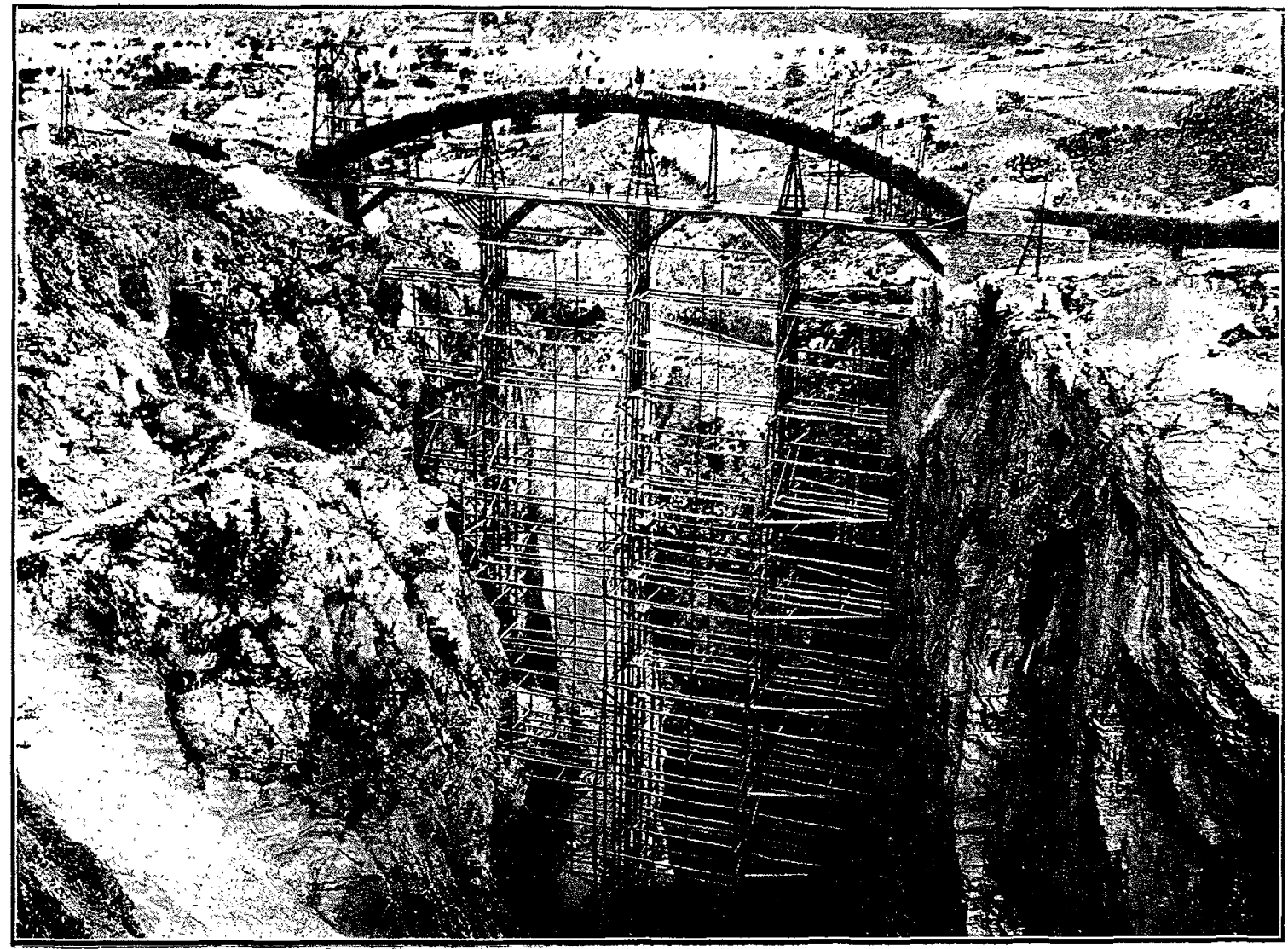

Lsine de Largentière-La Bessée (Hautes-Alpes)

Echafaudage nécessité pour le montage de la conduite-siphon surita Durance

Tourisme, M. Paul Michoud, président du Syndicat d'initiative de Grenoble et du Dauphiné, à Grenoble. - Participation étrangère, M. Pierre Chabert, président de la Section américaine des Conseillers du Commerce Extérieur de la France, à Paris.

Secrétaire général : M. A. BEAuQuis, secrétaire général de la Chambre de Commerce de Grenoble et de la XIIe Régión économique.

Secrétaire général adjoint: M. G. TochoN, secrétaire général de la Chambre Syndicale des Forces Hydrauliques, à Paris.

Directeur technique : M. MeuLey, ingénieur des Arts et Manufactures à Grenoble.

Conseiller technique : M. Auguste GIRY, ancien directeur adjoint au Commissaire général des Expositions coloniales de Marseille, 1906 et 1922 .

Service particulier de l'architecture : Architecte en chef, M. L. Jaussely, Grand Prix de Rome, architecte en chef du Gouver- c) Enfin, l'Etat apporte lui-même une contribution de trois millions.

Soit, en tout, 11 millions de capital initial.

Cette somme sera affectée à l'aménagement des terrains de l'Exposition, puis à la construction des grands palais et des nombreux édifices dont l'Exposition prendra directement la charge, enfin aux services d'administration, de publicité et propagande, etc..., etc... Les frais d'exploitation proprement dits seront couverts par les redevances payées par les exposants, les taxes perçues sur les attractions, le produit des entrées, la publicité concédée à l'intérieur de l'Exposition, etc...

\section{SON EMPLACEMENT.}

L'Exposition est installée dans de vastes terrains militaires désaffectés faisant partie du polygone du génie de la place de Grenoble, couvrant une surface de 20 hectares environ et situés 
aux portes mêmes de la ville de Grenoble, à 5 minutes à peine de la place Grenette et de la place Victor-Hugo, qui sont les deux principaux centres d'attraction et d'animation de la ville, et à 10 minutes environ de la gare P.-L.-M,

\section{L'entrée principale est desservie :}

10 Par la ligne de tramways actuelle de Grenoble à Eybens ;

20 Par une ligne de tramways spécialement établie pour le service de l'Exposition, partant de la gare P.-L.-M. comportant un tracé circulaire et à fonctionnement ininterrompu.

Dans l'esprit des pouvoirs publics qui ont conçu et réalisé l'Exposition, elle doit demeurer à titre de pare définitif qui deviendra le centre d'un quartier nouveau de Grenoble, et elle se trouve ainsi étroitement lièe à l'extension et à l'embellissement de la cité. çais : Compagnie Electro-Mécanique, Socièté des Ateliers de Construction Lyon-Dauphiné, Constructions Electriques de France, Sociélé Alsacienne de Constructions Mécaniques, Compagnie Thomson-Houston, Jeumont, Schncider, Sociélé Générale de Force et Lumière, Société des Machines à puissance autoréglable, Société 1)auphinoise Electrique, Société des Moteurs "Lilliput ", et par nos grands construcleurs de turbines : Neyret, Beylier \& Piccard-Pictet, Leflaive \& Cie, Constructions Electriques de France, Teisset, Rose \& Braul, Bouvier, Paul \& Cie, Magnat-Simon, Crozet-Fourneyron, elc...; pour faire ressortir les progrès réalisés dans la technique de la construction des machines hydro-électriques et thermiques, el dans le matériel appareillage nécessaire. Les Elablissements Merlin \& Gerin et les Ateliers de Delle exposent des postes complets a $120.000 \mathrm{v}$., et l'on peut voir fonctionner des compensateurs synchrones de grosses puissances relevant le facteur de puissance du réseau, des fours électriques, des redresseurs à vapeur de mercure à petit et

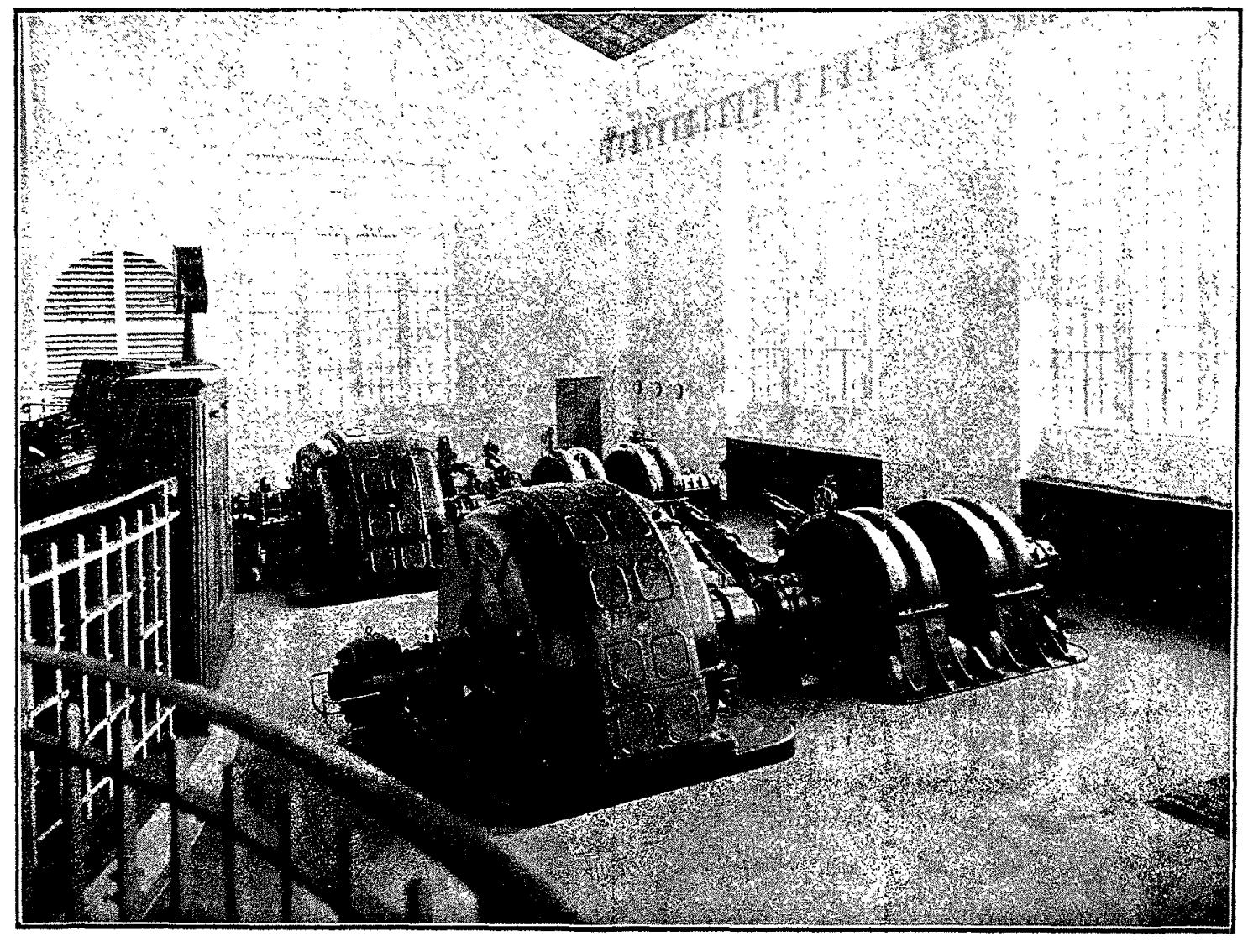

Usine de la Viclaire (Savoie)

Groupes de 120.000 chevaux - Compagnie Electro-Mécanique - Neyret-Beylier et Piccard-Pictet

\section{Section de la Houille Blanche.}

Dans l'ordre " Houille Blanche ", l'Exposition comprend :

$1^{\circ}$ Un grand Palais de la Houille Blanche, de 3.500 mètres carrés, à l'intérieur duquel est aménagé tout ce qui concerne plus spécialement la technique des hautes et basses chutes, savoir : l'équipement hydraulique des torrents et rivières, les conduites forcées et le matériel de chaudronnerie correspondant, les turbines et leurs régulateurs, les dynamos, les transformateurs et tous les appareils mécaniques connexes, les lignes de transport à toutes tensions et l'appareillage des réseaux, le matériel permettant l'utilisation sur place de l'énergie électrique par les procédés de l'électrochimie et de l'électrométallurgie et, d'une façon générale, tout ce qui concerne la production, l'utilisation immédiate et le transport à distance de l'énergie électrique.

Ce Palais est réservé à l'Industrie française de la Houille Blanche. Un effort. considérable a été fait par les constructeurs fran- à grand débit, c'est-à-dire les machines les plus perfectionnées que l'on trouve dans les installations modernes.

En ce qui concerne la technique de l'équipement des chutes, les projets de barrage, l'électrochimie et l'électrométallurgie, les plans, maquettes, machines, échantillons exposés par la Chambre Syndicale des Forces hydrauliques, les différents syndicats et industriels, sont de véritables merveilles.

Au-devant du Palais, des vasques superposées forment des successions de cascades qui se transforment, le soir, en fontaines lumineuses du plus bel effet; un immense bassin reçoit l'énorme volume d'eau débité par les cascades. Il est entouré de petits bassins avec jets rappelant les heureuses dispositions des grands jardins à la française.

$2^{\circ}$ Un Palais du Matériel des Chemins de fer, vaste hall de 90 mètres de longueur, qui abrite l'exposition des locomotives électriques du dernier modèle, les voitures de luxe les plus nou- 
velles, la présentation des signalisations en usage dans nos grands réseaux, etc...;

$3^{\circ}$ Une maison moderne d'habitation, comprenant toutes les utilisations ménagères et urbaines de l'électricité ; $5^{\circ}$ Un Pavillon des Arts généraux spécialement affecté aux peintres de la montagne et aux arts sportifs;

$6^{\circ}$ Un Pavillon des Grands Réseaux, exposition collective du plus haut intérêt, qui montre les ressources exceptionnelles apportées par les chemins de fer français à la prospérité économique du pays;

$7^{\circ}$ Un Bureau de Renseignements où seront délivrés les billets nécessaires et toutes indications relatives aux voyages pour toutes destinations, avec bureau de change, interprêtes, etc...;

$7^{\circ}$ Une Section organisée par les Eaux et Forêts et le T. C. F. et comprenant des chalets où seront présentés les travaux de cette Administration, des échantillons de la faune et de la flore alpine, avec pavillons de chasse, de pêche, aquarium, etc...;

$9^{\circ}$ Un Village Haut-Alpin, reconstitution pittoresque d'un des bourgs les plus élevés de nos Alpes avec sa population montagnarde.

Enfin, à l'axe même des deux sections, s'élève la Tour d'Orientation, audacieux monolythe en ciment armé, de 80 mètres de haut, sur laquelle est installée une table d'orientation correspondant au panorama incomparable qui permet d'embrasser, d'un seul coup d'œil, les admirables sommets des Alpes. $4^{\circ}$ Une ferme modèle pour la démonstration de toutes les applcations de l'électricité à l'exploitation agricole moderne.

\section{Section du Tourisme.}

Dans l'ordre touristique, l'Exposition comprend :

$1^{0}$ Un Palais du Tourisme réservé aux grandes organisations tcuristiques de France : Office National du Tourisme, TouringCub de France, Club Alpin Français, Artomobile-Club de France, etc..., Syndirats d'Initiative et Fédération de ces Syndicats, ainsi que l'Hôtellerie, stations thrmales et climatiques ;

¿o Un Palais des Transports et Industriss touristiques d'hiver et d'été ouvert. au multiples articles de sport et de voyage, à l'ameublement et à l'aménagement des hôtels, chalets et refuges, ainsi qu'aux cars-alpins, autobus, avions de voyage, etc...;

$3^{\circ}$ Un Palais du Tourisme 'd'autrefois, ol sont groupés tous les éléments de doclmentation rétrospective constituant l'iistoire des débuts et du développement du tourisme en France;

10 Un Palais du Tourisme colonial où no; possessions d'outre-mer ont mis en relief, de saisissante fạn, toutes les merveilles naturelles, les sites réputés et les vetiges d'antiquités illustres qui attirent vers elles le grand tourisme mondial.
Salles de RÉunions et de Congrès, Spectacles, Tour Belvédère, Atrractions diverses, Restauration, DégusTATION, ETC.

L'Exposition comprend encore :

$1^{\circ}$ Des salles de Congrès et de réunions qui seront des dépendances immédiates des grands palais ;

$2^{\circ}$ Un kiosque à musique pour les concerts journaliers et un théâtre en plein air, de 1.500 places, disposé aussi pour des repré-

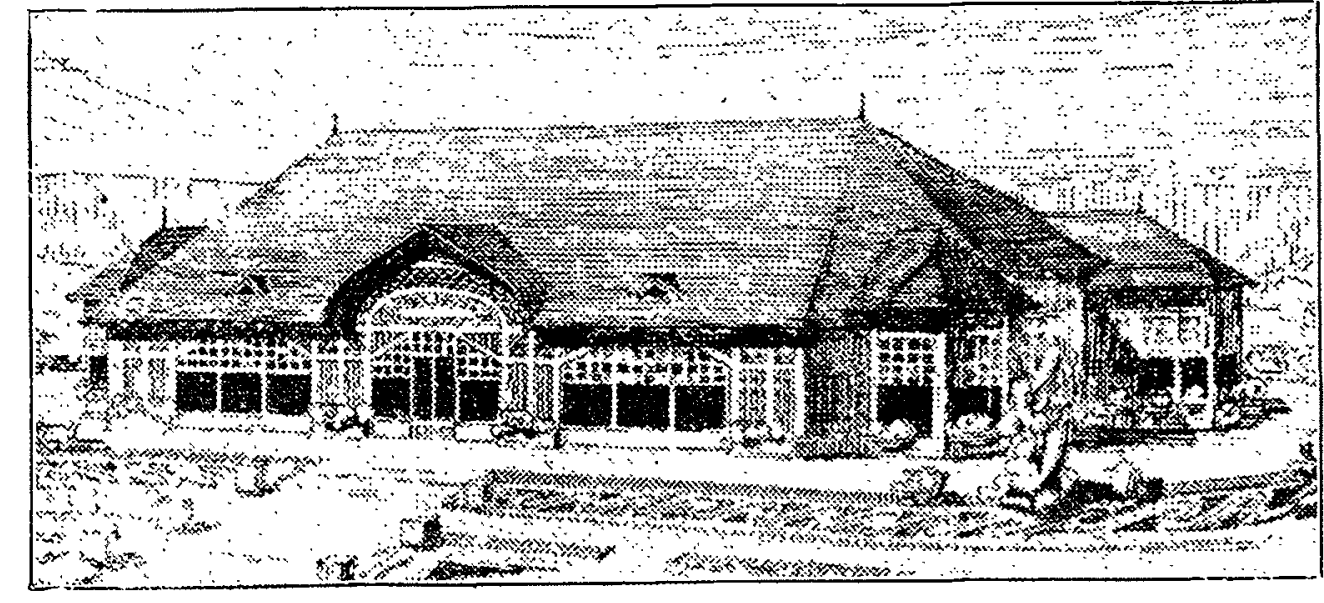

Grand Restaurant

sentations - cinématographiques, au cours desquelles, à côté du répertoire ordinaire de ce genre de spectacle, seront présentés des films rappelant aux spectateurs toutes les phases successives de la formation naturelle, de la transformation en énergie et de 
l'utilisation pratique de la Houille Blanche, film Oddoux-Sylvestre "Au Pays de la Houille Blanche ", puis tout ce qui concerne la vie touristique en France et dans nos colonies;

$3^{3}$ Un restaurant populaire, un restaurant de luxe et des stands spéciaux de dégustation de divers produits alimentaires;

40 Enfin toute une série d'attractions diverses, destinées à amuser, à attirer et à retenir certaines catégories de visiteurs.

L'Exposition Internationale de la Houille Blanche et du Tourisme de 1925 est la plus complète et la plus importante manifestation du genre qui ait été organisée à ce jour dans le monde entier.

A ce titre déjà elle doit, dans le domaine de la Houille Blanche, attirer l'attention des savants, des techniciens de l'électricité et des industriels de tous les pays.

Elle sera l'occasion de la réunion à Grenoble de nombreux Congrès scientifiques et industriels et, de ce fait, son rayonnement sur le monde économique sera considérable.

Voici le calendrier des Congrès inscrits :

Juin. - 12, 13, 14, Congrès de la Fédération des Coopératives P.-L.-M.

$15,16,17,18,19,20$ et 21 , Congrès de l'Association provinciale des architectes français.

20,21, Congrès de la Société de retraites pour les deux sexes P.-L.-M.

22, 23, 24, Office des Transports des Chambres de Commerce du Sud-Est.

$25,26,27$, Congrès national des vétérinaires.

28, 29, 30, Congrès du Groupe d'Etudes de la mobilisation économique, colonel Micanel.

Juillet. - 4, 5, 6, 7 et 8 , le matin, Congrès de la Houille Blanche organisé par la Chambre Syndicale des Forces hydrauliques.

Une journée commune aux deux Congrès.

$8,9,10,11$, Congrès des producteurs et distributeurs d'énergie.

8 au 11, Congrès des Anciens Elèves de l'Institut Electrotechnique de Grenoble.

12 et 13, Congrès des Ecoles Nationales professionnelles.

$20,21,22,23,24$, Congrès de la Société pour l'aménagement des eaux.

$22,23,24,25,26,27,28,29,30$, Congrès forestier international organisé par l'Administration des Eaux et Forêts et le Touring-Club de France.

27, 28, 29, 30 et $1^{\text {er }}$ août, Congrès de l'Association Française pour l'avancement des sciences.

Septembre. - 6, 7, 8, 9, Congrès de la Société des Anciens

Elèves des Ecoles Nationales des Arts et Métiers.

13, Congrès de la Fédération des Anciens Elèves des Ecoles Professionnelles et Ecoles pratiques.

$27,28,29,30$, Congrès de la Natalité.

Nota. - Il sera organisé par le Comité National de l'Union des Syndicats d'Ingénieurs une "Journée de l'Ingénieur ". La date n'est pas encore fixée.

Dans l'ordre du tourisme, elle attirera également l'attention de tous ceux qui, dans toutes les nations civilisées, ont compris que "l'amour des voyages", qui était resté jusqu'ici l'apanage des élites, pénétrait rapidement au plus profond de toutes les classes de la société, qu'il devenait un véritable besoin des peuples, et qu'il y avait lieu, par suite, de songer à fonder sur l'exploitation rationnelle et judicieuse de ce besoin, nouvel élément de bonheur de l'humanité tout entière, une bonne part de la prospérité des nations qui, à un titre quelconque, ont été dotées par la nature de beautés et de curiosités naturelles diverses.
Des Congrès, d'importantes réunions de tous les organismes de tourisme sont également envisagés ; de ce lait encore, elle aura un puissant retentissement et exercera un rayonnement incontestable sur le monde entier.

A côté de ces facteurs directs, bien faits déjà pour assurer à eux seuls le succès le plus complet à l'Exposition In ternationale de la Houille Blanche et du Tourisme de 1925, il convient de tenir compte des 200.000 touristes qui, chaque année en été, viennent visiter la région des Alpes et y séjournent plus ou moins longtemps.

Tous ceux de ces visiteurs qui sont en provenance du Centre, de l'Ouest, du Sud-Ouest et du Midi de la France, passent actuellement déjà par Crrenoble.

Il en est de même de ceux, très nombreux aussi, qui nous viennent de l'Algérie, de la Tunisie, du Maroc, comme aussi des étrangers qui débarquent à Bordeaux, et même à Nantes, et dans les ports méditerranéens.

L'Exposition de Grenoble, qui a été inaugurée officiellement le $21 \mathrm{mai}$, par M. Paul Painlexé, Président du Conseil, exercera sur tous ces touristes un attrait certain, ne manquera pas de les retenir au passage et pendant plusieurs jours.

En ce qui concerne les autres, ceux qui se rendent dans les stations balnéaires et climatiques de la Savoie et de la Haute. Savoie, par Aix-les-Bains et Annecy, ou par Bellegarde et Annemasse, on peut d'ores et déjà être certains que le rayonnement de l'Exposition Internationale de Grenoble ne manquera pas de s'exercer sur eux, et qu'ils seront invinciblement attirés vers cette puissante manifestation économique se déroulant tout près d'eux et où ils pourront contempler, dans un cadre magnifique et grandiose, les produits les plus utiles du labeur et de l'intelligence des hommes, à côté des beautés et des curiosités engendrées un pel partout au cours des siècles primitifs par les forces éternelles et toutes puissantes de la nature.

Au surplus, il a été mis en exploitation des services spéciaux par voie ferrée ou par autocars destinés à amener rapidement à Grenoble et vice-versa tous les touristes qui séjourneront dans les régions de Thonon-les-Bains, Evian-les-Bains, Chamonix, Saint-Gervais-les-Bains, Annecy, Aix-les-Bains, Moûtiers-Salins, Chambéry, Allevard-les-Bains, etc...

Enfin, l'organisation de l'Office du Logement, place de la Gare (Tél. 20-13), assure à tous les visiteurs des chambres à des prix convenables. Nous ne saurions trop encourager les nombreix lecteurs de notre Revue à venir à Grenoble à l'occasion de cete grandiose manifestation; ils peuvent être certains d'y trouvel le meilleur accueil.

Troisième Congrès de la Houllle Blanche.

Le troisième Congrès de la Houilie Blanche organisé ṕar la Chambre syndicale des Forces hydrauliques, de l'Electrochimit, de l'Electrométallurgie et des Industries qui s'y rattachent, aura lieu à Grenoble, du 4 au 8 juillet 1925.

Il y a vingt-trois ans que, sous les auspices du Syndicat dss propriétaires et industriels possédant ou exploitant des forces motrices, s'ouvrait à Grenoble, le 7 septembre 1902, le premibr Congrès de la Houille Blanche. Ce premier Congrès, qui eut in retentissement considérable, fut sous la présidence d'honneur de M. Guillain, ancien Ministre des Colonies, Inspecteur généal des Ponts et Chaussées, et de M. Hanotaux, ancien Ministre les Affaires étrangères, membre de l'Académie Française; sous la présidence effective de $M$. Charles PINAT, ancicn Ingénieur des Ponts et Chaussées, Maitre de forges à Allevard. L'un des seré- 
taires était le regretté fondateur de notre Revue, M. Cote, Ingénieur électromélallurgiste.

La Chambre Syndicale des Forces hydrauliques, de l'Electrochimie, de l'Electrométallurgic et des Industries qui s'y rattachent, organise, comme nous venons de le dire, à Grenoble, des 4 au 8 juillet prochain, à l'occasion de l'Exposition, le troisième Congrès International de la Houille Blanche. Ce troisième Congrès metlra en lumière tous les progrès accomplis au cours de ces dernières années, dans le domaine de la Houille Blanche; il permellra de mesurer ce qui reste encore à faire et hâter dans toute la mesure du possible, aussi bien dans l'ordre scientifique que dans l'ordre économique, la réalisation de rapides et décisifs progrès.

LéCongrès, auquel les Pouvoirs publics prètent largement leur appui, aura un caractère international, la Chambre Syndicale des Forces hydrauliques devantinviter les représentants les plus éminents des principaux pays producteurs de houille blanche.

Il sera ouvert le 4 juillet; sa durée sera de 4 jours.

En raison de l'intérêt national présenté par cette manifestations, les Compagnies de chemins de fer françaises ont décidé d'accorder exceptionnellement aux membres titulaires du Congrès une réduction de $50 \%$. Line circulaire ultérieure sera d'ailleurs adressée aux adhérents après réception de leur adhésion et donnera tous les renseignements utiles pour l'obtention de ce demi-tarif.

Nos lecteurs trouveront ci-après :

$1^{\circ}$ La composition du Comité de Patronage et de la Commission d'organisation du Congrès ;

$2^{\circ}$ La composition des bureaux de sections el des sous-sections, la nomenclature des rapports, le programme des questions qui sont soumises aux délibérations du Congrès ;

\section{$3^{\circ}$ Le règlement général du Congrès.}

Les articles 5 et 7 du règlement général indiquent les cond-i tions à remplir pour participer au Congrès, soit comme membre titulaire, soit comme membre associé.

Le Comité compte, en outre, organiser dans la vallée du Grési- vaudan, une excursion qui aura pour objet de glorifier les ancêtres de la Houille Blanche.

\section{Composition du Comité de Patronage et de la Commission d'Organisation.}

Sous le haut patronage de : M. Doumergue, Président de la République,

Et la Présidence d'honneur de : MM. Loubet, ancien Président de la République ; Fallières, ancien Président de la République; PorNCARÉ, ancien Président de la République; Millerand, ancien Président de la République ; M, le Président du Conseil des Ministres, les Ministres des Affaires étrangères, des Travaux Publics, des et de l'Agriculture.

\section{CoMItÉ}

D'ORGANISATION.

\section{A Paris :}

Président : M. Louis Marlio, Président de la Chambre Syndicale des Forces hydrauliques. - Vice-présidents : MI. Barct. Bouchayer, Gall, Pinot. - Trésorier : M. Charpenay. Secrétaire général : M. Tochon. - Secrétaire général adjoint : M. Meynial. - Membres : MM. Bachellery, Boltgault, de la Brosse, Eydoux, Flusin, Mathieu, Margot, Maroger, Rateau.

\section{A Grenoble :}

Président: M. Aimé BOUCHAYER- Secrétaire général: M. Maurice Bouchayer. Membres:MM. CHARPENAY, FrEDET, KeLler, LaCrotX, LÉPINe, PERINEL, L. ROCHETTE.

\section{Composition}

DES BUREAUX DES SECTIONS ET LISTE Des Rapports.

\section{SECTION}

ADMINISTRATIVE.

Président : M. R. DE LA Brosse, Inspectem général des Ponts et Chaussées, 87, boulevard du Montparnasse, Paris. - Vice-président : M. Aimé Bouchayer, Industriel, vice-président de la Chambre de Commerce de Grenoble; président de l'Association des Producteurs des Alpes Françaises, 34 bis, boulevard Gambetta, à Grenoble (Isère). - Secrétaire : M. WeBER, docteur en droit, 7 , rue de Madrid, à Paris. 
$1^{\text {er }}$ Rapport. - Améliorations à apporter aux lois ou règlements qui régissent les rapports de l'Etat et des Sociétés ou particuliers qui projettent d'équiper ou qui exploitent une chute d'eau ou une ligne de transport à haute tension.

Rapporteur : M. Bougault, avocat à la Cour d'Appel de Lyon, président du Comité du Contentieux de la Chambre Syndicale des Forces hydrauliques, 10, rue Vaubecour, à Lyon (Rhône).

2e Rapport. - Les charges fiscales qui pèsent sur les Sociétés ou particuliers concessionnaires d'une chute d'eau ou d'une ligne de transport à haute tension.

Rapporteur : M. Threrry, directeur général de l'Energie électrique du Sud-Ouest, 180, boulevard Antoine-Gautier, à Bordeaux

$3^{\mathrm{e}}$ Rapport. - Améliorations à apporter dans les rapports des Sociétés ou particuliers qui projettent d'équiper ou qui exploitent une chute d'eau ou une ligne de transport à haute tension avec les tiers propriétaires de terrains, possesseurs de droits d'eau ou précédents usagers (permissionnaires ou concessionnaires).

Rapporteur : M. Achille Mestre, professeur à la Faculté de Droit de Paris, 106, boulevard Saint-Germain, Paris.

$4^{\mathrm{e}}$ Rapport. - Améliorations des conditions de vente ou de transport d'énergie imposées aux concessionnaires de chute d'eau ou de réseaux à haute tension au profit du public.

Rapporteur : M. Devun, directeur de la Compagnie Electrique de la Loire et du Centre, administrateur-délégué de la Société de Transport d'Energie du Centre, 56, rue du Faubourg-SaintHonoré, Paris.

$5^{\mathrm{e}}$ Rapport. - Fixation et mise à disposition des réserves en eau et en force imposées aux concessionnaires de chutes d'eau.

Rapporteurs : MM. Perrin, ingénieur au Corps des Mines, directeur des Aciéries d'Ugine, à Ugine (Savoie); DE Maublanc, ingénieur civil des Mines, attaché aux Services hydrauliques de la Société d'Electrochimie, d'Electrométallurgie et des Aciéries électriques d'Ugine, à Ugine (Savoie).

$6^{\text {e }}$ Rapport. - Les règles de droit international concernant l'utilisation de la force motrice des cours d'eau ou des lacs appartenant à deux pays ou limitrophes entre deux pays.

Rapporteur : M. GÉnissiev, ingénieur en chef des Ponts et Chaussées, 22, rue du Regard, Paris.

7e Rapport. - Réglementation de l'exportation et de l'importation de l'énergie électrique.

Rapporteur : M. Aimé Bouchayer, administrateur-délégué des Etablissements Bouchayer \& Viallet, président de l'Association des Producteurs des Alpes Françaises, 34 bis, boulevard Gambetta, à Grenoble.

$8^{\mathrm{e}}$ Rapport. - Les caractères juridiques de la concession d'énergie hydraulique.

Rapporteur : M. Pepr, professeur à la Faculté de Droit de Grenoble, à Grenoble.

\section{SECTION ÉCONOMIQUE ET FINANCIÈRE.}

Président : M. Albert Mathieu, sénateur du Nord, inspecteur général des Ponts et Chaussées, secrétaire général honoraire du Ministère des Travaux publics, 70 bis, rue d'Amsterdam, Paris. Vice-présidents : MM. CELIER, ancien conseiller d'Etat, directeur général honoraire au Ministère des Finances, administrateur du Comptoir National d'Escompte de Paris, 55, rue de Babylone, Paris; Wibratte, directeur de la Banque de Paris et des PaysBas, 3, rue d'Antin, Paris. - Secrétaire : M. LECAT, ingénieur des Ponts et Chaussées, 244, boulevard Saint-Germain, Paris.

1 er Rapport. - La Houille Blanche dans l'économie nationale en France et à l'étranger.
Rapporteur : M. Raoul Blanchard, professeur à la Faculté des Letlres de Grenoble, 3, rue Très-Cloître, à Grenoble (Isère).

$2^{\mathrm{e}}$ Rapport. - Quelques indications sur le prix de revient de l'énergie éleclrique de provenance hydraulique.

Rapporteur : M. Gustave Mrncrer, ingénieur en chef des Ponts et Chaussées, administrateur-délégué de l'Union Hydro-Electrique, ingénieur-conseil de l'Union d'Electricité, 3, rue Christophe Colomb, Paris.

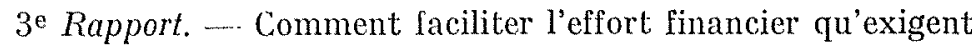
la mise en valeur de nos chutes d'eau, le transport et la meilleure utilisation de l'énergie qui en provient.

Rapporteurs : MM. BLoch, administrateur-délégué de la Banque Transatlantique, 10, rue Mogador, Paris: Maroger, président de l'Ĺnion des Producteurs des Pyrénées Occidentales, 19, rue Louis-le-Grand, Paris.

$4^{\mathrm{e}}$ Rapport. - Les conditions de la concurrence entre l'énergie de provenance hydraulique et l'énergie de provenance thermique.

Rapporteur : M. Arbelot, directeur honoraire des Forces hydrauliques et des Distributions d'Energie électricue au Ministère des Travaux publics, directeur général de la Société des Grands Travaux de Marseille, 25, rue de Courcelles, Paris.

$5^{\mathrm{e}}$ Rapport. - Avantages économiques résultant de l'interconnexion des usines génératrices.

Rapporteur : MI. Duval, directeur des Services électriques à la Société Générale d'Entreprise, 56, rue du Faubourg-SaintHonoré, Paris.

\section{Section technigue.}

Président : M. Rateau, industriel, membre de l'Institut, 40, rue du Colisée, Paris. - Vice-président : M. Margot, directeur général de la Compagnie des Chemins de fer de Paris à Lyon et à la Méditerranée, 88, rue Saint-Lazare, Paris. - Adjoint au président : M. Eydoux, professeur à l'Ecole Nationale des Ponts et Chaussées 3 , rue Théodore-de-Banville, Paris. - Secrétaire : M. Dupin, ingénieur des Ponts et Chaussées, 97 bis, rue Notre-Dame-desChamps.

\section{I. - Sous-section de la Production.}

Président : M. Eypoux, professeur à l'Ecole Nationale des Ponts et Chaussées, 3, rue Théodore-de-Banville, Paris.

1er Rapport. - La conjugaison des usines et l'utilisation des réservoirs pour régulariser la tension .

Rapporteur : M. Leclerc du SABlon, ingénieur des Ponts et Chaussées, ingénieur en chef de la Compagnie du Midi (Service de la construction des usines hydroélectriques), 7, rue de Dalbade, à Toulouse.

$2^{\text {e }}$ Rapport. - Les apports solides des cours d'eau et l'engravement des prises d'eau et des réservoirs.

Rapporteurs : MM. Simon, ingénieur en chef des Ponts et Chaussées (Service des forces hydrauliques de la région du SudEst), 6, rue Haxo, Grenoble (Isère); Hatgelen, ingénieur des Ponts et Chaussées, 6, rue Haxo, à Grenoble.

$3^{\text {e }}$ Rapport. - Les principes théoriques et pratiques de la construction des grands barrages. Les accessoires des grands barrages, déversoirs, vannes, siphons.

Rapporteur : M. Degove, ingénieur en chef des Ponts et Chaussées (Service spécial d'aménagement de la Haute Dordogne), 1, place Valhubert, à Paris.

$4^{e}$ Rapport. - Les usines sur fleuves : Rhône, Rhin, barrages à vannes et automatiques.

Rapporteurs : MM. Louis Vennin, ingénieur en chef des Poudres, directeur général de la Compagnie Générale d'Entreprises, 
56, faubourg Saint-Honoré, Paris ; Delaly, ingénieur E. C. P., chef de service de l'Entreprise des travaux de l'usine de PougnyChancy, 56, rue du Faubourg-Saint-Honoré, Paris.

$5^{\mathrm{e}}$ Rapport. - Les canaux d'amenée et les cheminées d'équilibre.

Rapporteurs : MM. Eydoux, professeur à l'Ecole Nationale des Ponts et Chaussées, 3, rue Théodore-de-Banville, Paris; GouA, ingénieur des Arts et Métiers, Etudes d'hydraulique et de Génie civil, 29, rue d'Artois, Paris.

$6^{\mathrm{e}}$ Rapport. - Les pertes de charges singulières dans les conduites forcées.

Rapporteur : M. Auguste Bouchayer, ingénieur des Arts et Manufactures, Société Hydro-électrique "Drac-Romanche ", 155, cours Berriat, Grenoble.

7e Rapport. - L'évolution des turbines.

Rapporteur : M. Chalons, ancien élève de l'Ecole Polytechnique, 19, rue Louis-le-Grand, Paris.

80 Rapport. - Les accessoires des turbines, pivots, robinets, régulateurs, raccords des turbines et des conduites.

Rapporteur : $\mathrm{M}$.

Maurice Gariel, directeur général des Ateliers Neyret, Beylier et Piccard-Pictet, boîte postale No 52 , à Grenoble.

9 e Rapport. - Les essais du matériel des usines hydrauliques.

Rapporteur : $\mathrm{M}$. LAURENT, ingénieurconseil, chargé des essais extérieurs de la Société Hydrotechnique de France, 116 , avenue d'Orléans, Paris.

$10^{\mathrm{e}}$ Rapport.-. La Houille Bleue.

Rapporteur : M. DE Rouville, ingé-

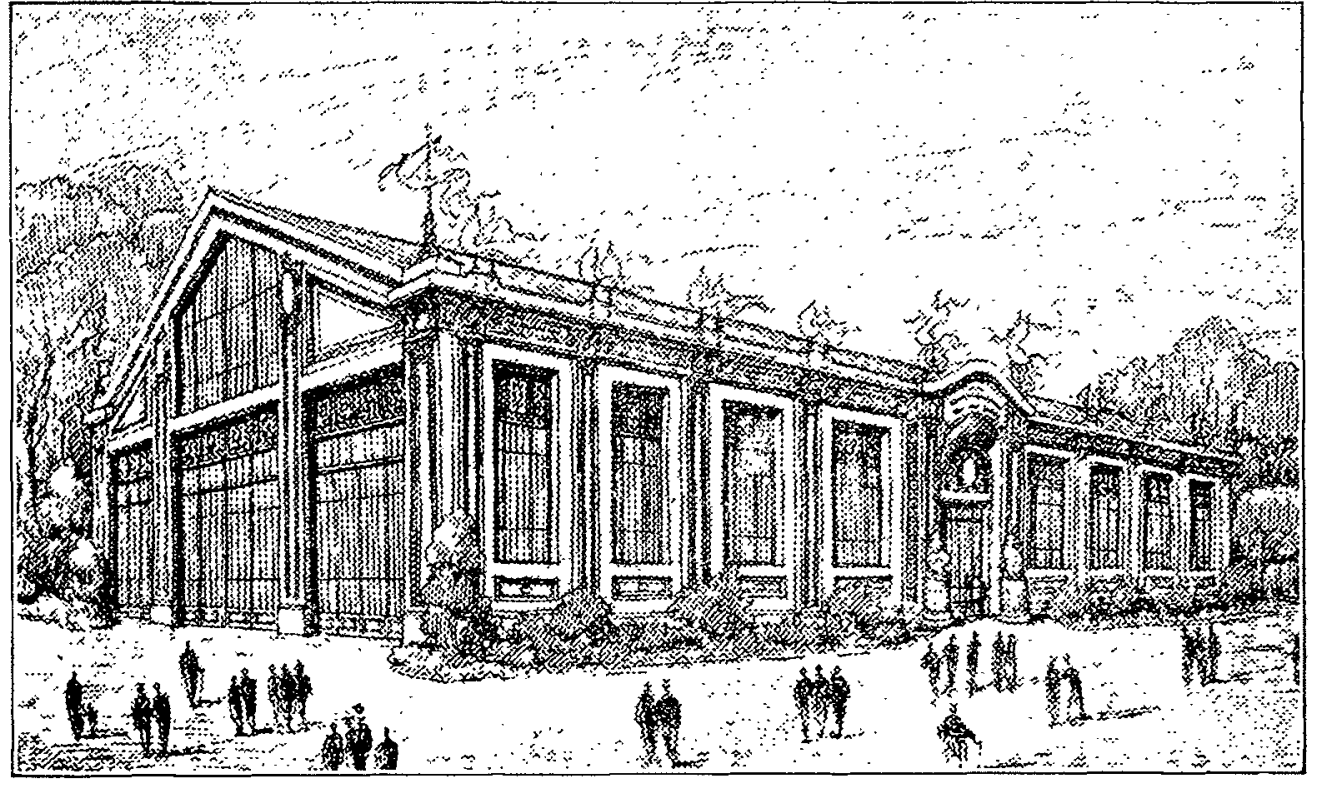

Palais des Chemins de fer $1^{\text {er }}$ Rapport. - Technique moderne des réseaux de transmission d'énergie à haute tension.

Rapporteur : M. LAVANCHY, docteur ès-sciences, ingénieur E. S. E., ingénieur à la Compagnie Générale d'Entreprises, 56, rue du Faubourg-Saint-Honoré, Paris.

$2^{\text {e }}$ Rapport. - Emploi de l'aluminium et de l'aluminium-acier pour conducteurs.

Rapporteur : M. Dusaugey, ingénieur civil des Mines, 4, place de Verdun, Grenoble.

$3^{\mathrm{e}}$ Rapport. - Exploitation des grands réseaux de transport, direction générale, liaisons téléphoniques et télégraphiques entre les divers réseaux, incidents et accidents d'exploitation.

Rapporteur : M. Laporte, ancien élève de l'Ecole Polytechnique, directeur de l'Union Hydro-électrique, 3, rue ChristopheColomb, à Paris.

Communication sur la liaison et le réglage des transports à haute tension, par M. Boissonnas, administrateur-délégué de la Société Générale de Force et Lumière, 3, rue de la Boétie, Paris. nieur en chef des Ponts et Chaussées, 43, avenue du PrésidentWilson, Paris.

11e Rapport. - L'emploi des modèles réduits pour l'étude des problèmes techniques d'hydraulique.

Rapporteur : M. Camichel, correspondant de l'Institut, professeur à l'Université de Toulouse, directeur de l'Institut Electrotechnique, 29 bis, rue Maignac, à Toulouse.

$12^{\mathrm{e}}$ Rapport. - Contribution à l'étude de I'hydrologie industrielle.

Rapporteur : M. Coutagne, ingéniemr en chef des Services hydrauliques et électro-métallurgiques de la Société des Aciéries et Forges de Firminy, 1, rue Chernoviz, à Paris (16e).

Communication sur l'évaluation de l'énergie des installations hydrauliques, par M. A. Boucher, à Prilly, canton de Vaud (Suisse).

\section{II. - SOUS-SECTION DU TRANSPORT.}

Président : M. BAcheldery, ingénieur en chef du Matériel et de la Traction à la Compagnie des Chemins de fer du Midi, 54, boulevard Haussmann, à Paris.
III. - SOUS-SECTION $\mathrm{DE}$

L'UTILISATION.

Président : M. FLuSIN, professeur à la Faculté des Sciences de Grenoble, à Grenoble (Isère).

1 er Rapport.-Utilisation rationnelle au point de vue technique de la houille blanche pour l'électrification des chemins de fer.

Rapporteur : M. PARODI, ingénieur en chef des Services électriques à la Compagnie du Chemin de fer de Paris à Orléans, 141; quai d'Orsay, Paris.

$2^{\text {e }}$ Rapport - Utilisation de la puissance résiduelle dans le cours de la journée (puissance de nuit, puissance disponible pendant les heures creuses de la journée) et de la puissance permanente ou puissance des hautes eaux.

Rapporteur : M. Paul Bergeon, professeur à l'Institut Electrotechnique de Grenoble, 8, place Victor-Hugo, à Grenoble.

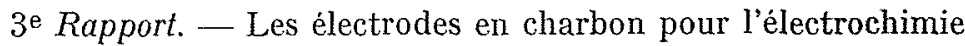
et l'électrométallurgie, l'électrode Soderberg.

Rapporteur : M. François GALL, directeur de la Société des Electrodes de la Savoie, à Notre-Dame-de-Briançon (Savoie).

4e Rapport. -- Les industries électrochimiques par voie humide.

Rapporteur : M. Dony-Henault, professeur à la Faculté des Sciences appliquées de l'Université de Bruxelles, membre correspondant de l'Académie Royale de Belgique, 30, rue Emile-Banning, à Bruxelles (Belgique).

$5^{\mathrm{e}}$ Rapport. - Les industries électrométallurgiques par voie humide, utilisation rationnelle de la houille blanche à ces industries.

Rapporteur : M. Altmayer,i ingénieur des Arts et Manufac- 
tures, Banque de Paris et des Pays-Bas, 41, avenue de l'Opéra, Paris.

$6^{\text {e }}$ Rapport. - Le carbure de calcium et la cyanamide calcide, utilisation ratiomelle de la houille blanche à ces industries.

Rapporteur : MI. Jean GalL, ingénieur des Mines, 5, rue Fabvre, à Chambéry (Savoie).

$7^{\mathrm{e}}$ Rapport. - Les industries électrothermiques des fontes, fers, aciers et des ferro-alliages : utilisation rationnelle de la houille blanche à ces industries.

Rapporteurs : MM. Matruiev, ancien élève de l'Ecole Polytechnique, ingénieur civil des Mines, directeur général des Services électrométallurgiques de la Société d’électrochimie, d’électrométallurgie et des Aciéries électriques d'Ugine, avenue de Chevène, à Annecy (Haute-Savoie); SrTer, ingénieur en chef des Services

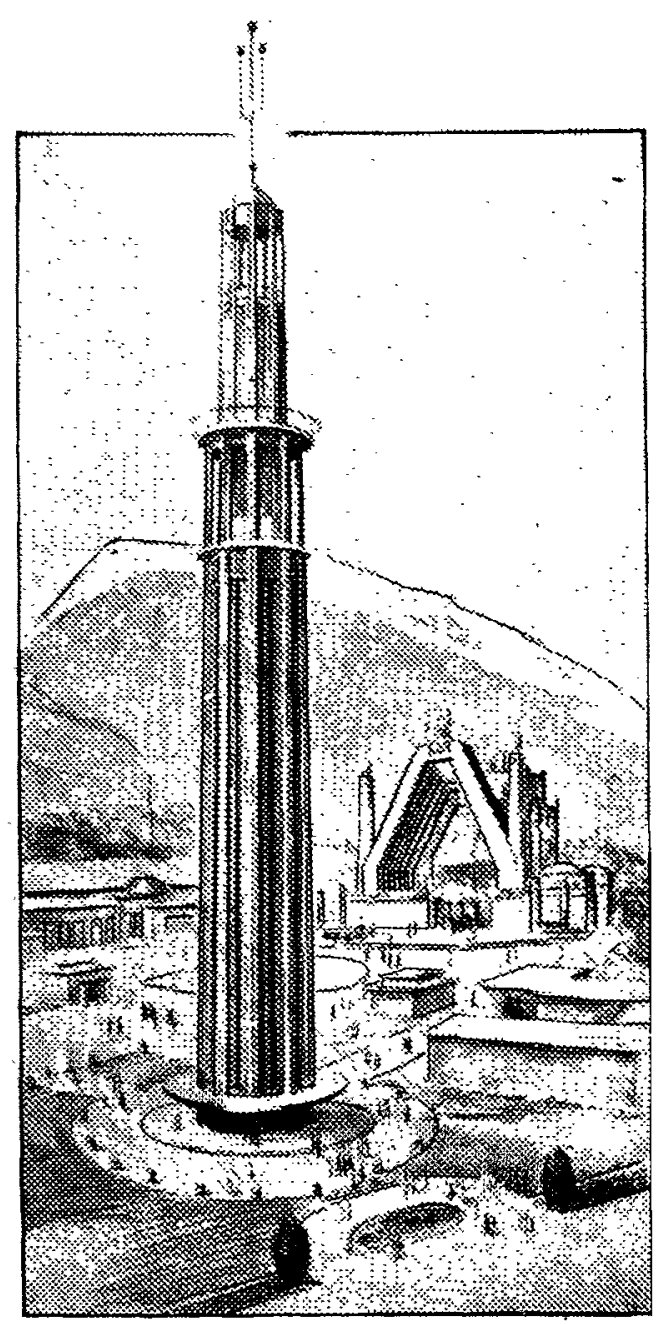

La Tour d'Orientation de 80 mètres

hydrauliques à la Société d'électrochimie, d'électrométallurgie et des Aciéries électriques d'Ugine, à Ugine (Savoie).

8e Rapport. - L'aluminium et les métaux légers : le rôle de la houille blanche dans ces industries.

Rapporteur : M. SuHr, ingénieur en chef, directeur des Services des recherches à la Compagnie des Produits chimiques et électrométallurgiques d'Alais, Froges et Camargue, 126, rue de la Boëtie, Paris.

9e Rapport. - L'industrie électrothermique du zinc.

Rapporteur : M. Canaud, chef des travaux de chimie à la Faculté des Sciences de Grenoble, à Grenoble (Isère).
NOTICES (1)

$1^{\circ}$ Le laboratoire de Beauvert;

$2^{\circ}$ Les installations hydrauliques de la vallée de la Pique;

$3^{\circ}$ Les installations hydrauliques de la vallée d'Ossau;

$4^{\circ}$ Les installations hydrauliques de la vallée de Beaufort (Savoie) ;

$5^{\circ}$ L'aménagement du Rhône;

$6^{\circ}$ L'aménagement du Rhin ;

$7^{\circ}$ Le barrage et l'usine du Blavet;

80 Le barrage el l'usine d'Eguzon;

$9^{\circ}$ L'usine de la Viclaire ;

$10^{\circ}$ L'usine de la Rhue;

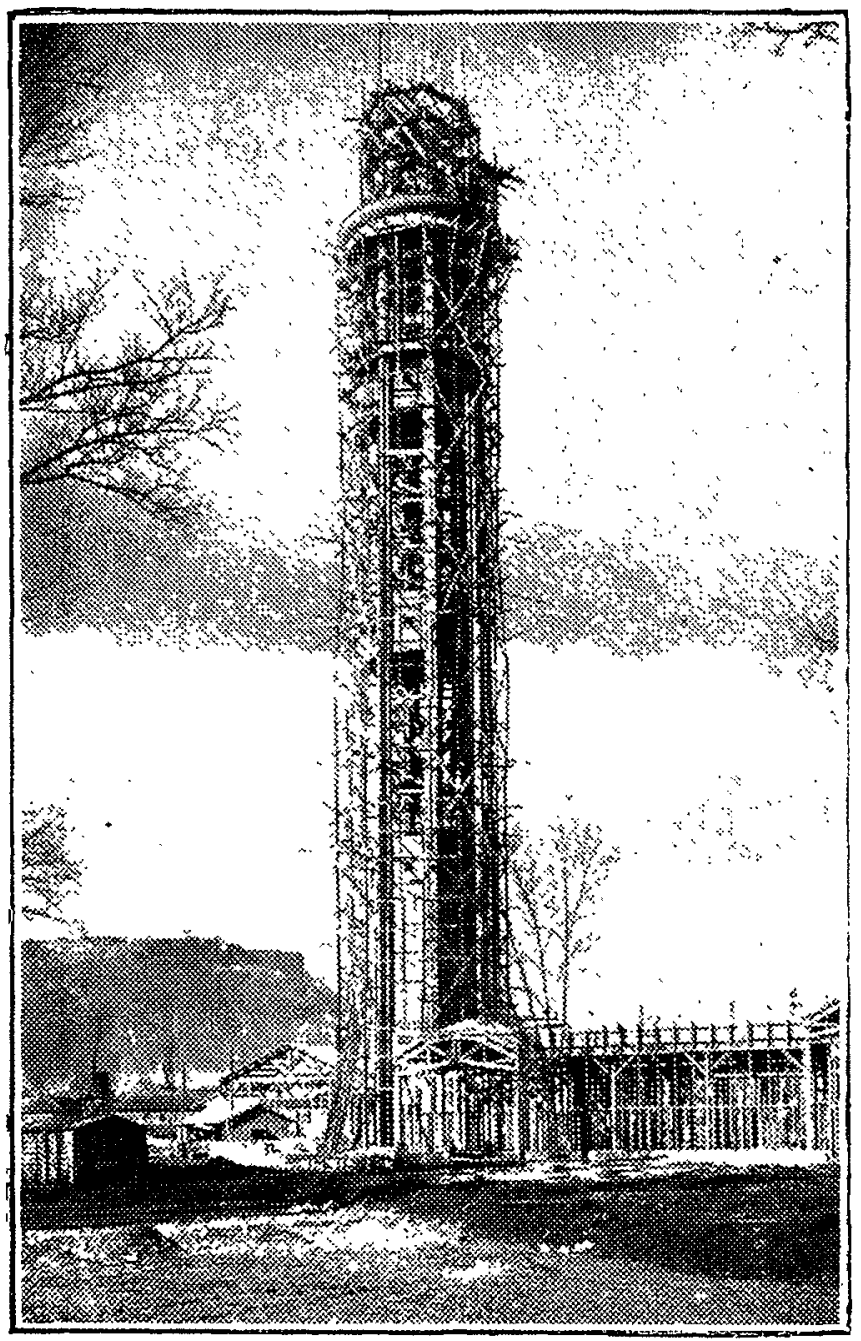

La Tour d'Orientation en construction

$11^{\circ}$ L'usine de Chancy-Pougny;

120 La Société de Transport d'énergie des Alpes;

$13^{\circ}$ L'Union des Producteurs des Pyrénées-Occidentales;

$14^{\circ}$ Le transport de force Eguzon-Paris;

$15^{\circ}$ Le transport de force Phône-Jura;

$16^{\circ}$ L'électrification des chemins de fer du Midi;

$17^{\circ}$ L'électrification des chemins de fer du P.-L.-M. ;

$18^{\circ}$ L'électrification du chemin de fer du P.-O.

(1) Les notices seront publiées dans les volumes où paraîtront le compte-rendu du Congrès, les rapports et les vœux adoptés. 
$19^{\circ}$ L'hydro-électricité au Maroc;

$20^{\circ}$ L'usine de Yaté en Nouvelle-Calédonie.

\section{REGLEMENT}

Article premier. - Le troisième Congrès de la Houille Blanche se tiendra à Grenoble en 1925. Ce Congrès s'ouvrira le samedi 4 juillet, sa durée sera de quatre jours.

ArT. 2. - Le Congrès comprend trois sections : Une section administrative, une section économique et financière, une section lechnique.

La section technique est subdivisée en trois sous-sections : Sous-section I : sous-section de la production; sous-section II : sous-section du transport; sous-section III : sous-section de l'utilisation.

Art. 3. - Les séances auront lieu aux heures arrêtées par le Comité d'organisation.

Le Congrès comportera deux séances plénières, quatre séances de sections, une visite à l'Exposition de la Houille Blanche et du Tourisme, une excursion.

A la fin du Congrès se tiendra une séance commune à la Chambre Syndicale des Forces hydrauliques et au Syndicat professionnel des Producteurs et Distributeurs d'énergie électrique.

Les questions seront d'abord discutées en séances de sections, ensuite en assemblées plénières.

Les discussions sont ouvertes à chaque séance. Aucun membre ne peut occuper la tribune plus de quinze minutes, ni plus de deux fois dans la mème séance, à moins que l'assemblée n'en décide autrement.

Les orateurs sont priés de remettre au bureau de la section, dans les vingt-quatre heures, un résumé des paroles qu'ils auront prononcées.

Après discussion de chaque question, la section peut désigner un ou plusieurs rapporteurs chargés de soutenir en séarice plénière les conclusions qu'elle a adoptées.

AnT. 4. - Les membres du Congrès ont seuls le droit de présenter des travaux et de prendre part aux discussions.

ART. 5. - La cotisation pour prendre part au Congrès est fixée à 50 francs.

Cette cotisation donne de plus accès aux séances du Congrès et permet de prendre part aux réceptions et à l'excursion qui sera faite dans la vallée du Grésivaudan pour glorifier les ancêtres de la houille blanche.

Les frais d'excursion ne sont pas compris dans la cotisation, chaque membre ne payant que ceux de l'excursion à laquelle il prend part.

La cotisation donne droit en outre, pendant la durée du Congrès, à une carte permanente à l'Exposition de la Houille Blanche et du Tourisme.

Ces cartes sont strictement personnelles.

Art. 6. - Les rapports et vœux du Congrès seront réunis en deux volumes et publiés. Les membres du Congrès qui désireront recevoir ces volumes devront en faire la demande dès leur inscription. La souscription pour l'ensemble de ces publications est fixée à 130 francs.

Toutefois, les rapporteurs recevront gracieusement vingt-cinq exemplaires d'un tirage à part de leurs rapports.

ART. 7. - Pourront participer au Congrès, à titre de membres associés, les presonnes de la famille d'un membre ordinaire (femmes, enfants, frères, sœurs), moyennant le versement d'une cotisation dont le montant est fixé à 25 franes. Cette cotisation leur donnera droit aux avantages énumérés ci-dessus, exception faite cependant des séances du Congrès auxquelles les membres associés n'auront pas accès.

Art. 8. - Par dérogation à l'article 5, seront dispensés du paiement de la cotisation les membres des administrations publiques et délégués étrangers que le Bureau de la Chambre Syndicale des Forces hydrauliques aura cru devoir inviter.

ART. 9. - Les personnes qui désireront prendre part au Congrès devront faire parvenir leur adhésion et leur cotisation, au plus tard le 1 juin 1925, à M. G. Tochon, secrétaire général de la Chambre Syndicale des Forces hydrauliques, 7, rue de Madrid, Paris.

L'inscription sur la liste des participants du troisième Congrès à un titre quelconque, comporte l'adhésion aux dispositions du . présent règlement.

Art. 10. - Le Bureau de la Chambre Syndicale des Forces hydrauliques statue en dernier ressort sur tout incident qui n'auraitpas étéprévu au règlement.

Nous espérons que beaucoup de nos lecteurs voudront bien accorder leur précieux concours à la réusşite de ce Congrès, en envoyant leur adhésion à $M$. G. Tochos, secrétaire général de la Chambre Syndicale des Forces hydrauliques, 7, rue de Madrid, Paris. 